\title{
Stability Conditions of Zero Solution for Third Order Differential Equation in Critical Case
}

Thair Y . Thanoon

Saad Fawzi AL-Azzawi

saad_fawzi78@yahoo.com

Department of Mathematics, College of Computer Sciences and Mathematics, University of Mosul, Mosul, Iraq

\section{Received on: 20/05/2007}

\section{Accepted on: 28/06/2007}

\section{ABSTRACT}

In this paper, we study the conditions under which the zero solution is stable in the semi- liner case for certain third order differential equation of the form :

$$
y^{\prime \prime \prime}+p_{1}(t) y^{\prime \prime}+p_{2}(t) y^{\prime}+p_{3}(t) y=h\left(t, y, y^{\prime}, y^{\prime \prime}\right)
$$

Where

$$
\begin{gathered}
p_{s}=\pi^{s}\left[q_{s}+w_{s}(t)\right], \quad, \quad s=1,2,3 \quad w_{s}(t): \Delta \rightarrow C, \quad q_{s} \in C, \\
a \in N, \quad t \in \Delta=[a, \infty)
\end{gathered}
$$

The characteristic equation of the above differential equation has complex roots of the form :

and the other root has the following $\quad \lambda_{0}>0, \lambda_{1}=-\lambda_{2}=i \lambda_{0}$

$\operatorname{Re} \lambda_{3}<-M, M>0$ property

Keywords: Stability, Critical Case.

$$
\begin{aligned}
& \text { شروط استقرارية الحل الصفري لمعادلة تفاضلية من الرتبة الثالثة في احدى الحالات الحرجة }
\end{aligned}
$$

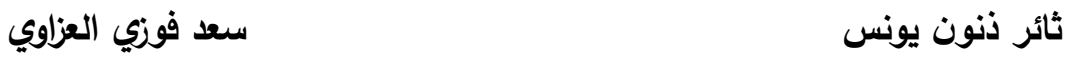

$$
\begin{aligned}
& \text { كلية علوم الحاسوب والرياضيات، جامعة الموصل }
\end{aligned}
$$

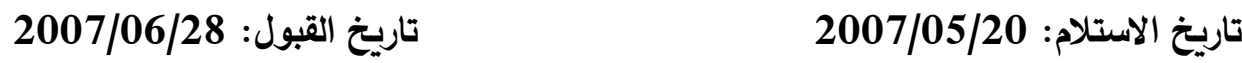

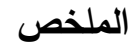

$$
\begin{aligned}
& \text { هذا البحث سيدرس شروط استقرارية الحل الصفري في الحالة شبه الخطية لدعادلة تفاضلية من } \\
& \text { الرتبة الثالثة بالثكل : } \\
& \text { حيث ان } \\
& p_{s}=\pi^{s}\left[q_{s}+w_{s}(t)\right] \quad, \quad \mathrm{s}=1,2,3 \quad w_{s}(t): \Delta \rightarrow C, \quad q_{s} \in C \quad \text {, } \\
& a \in N, \quad t \in \Delta=[a, \infty) \\
& \text { ان المعادلة المميزة للمعادلة التفاضلية اعلاه لها زوج من الجذور المعقدة بالثكل : }
\end{aligned}
$$

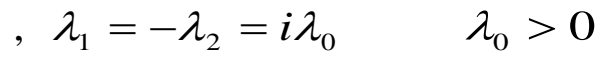

$$
\begin{aligned}
& \text { والجذر الاخر يحقق الخاصية Re } \\
& \text { الكلمات المفتاحية: الاستقر ارية, الحالة الحرجة }
\end{aligned}
$$




\section{1- INTRODUCTION}

Critical cases in the theory of stability for differential equation means, that cases when the real part of all roots of the characteristic equation are nonpositive with the real part of at least one root being zero , other express which is neither stable nor unstable [3] .

In the critical case the non-liner terms begin to influence the stability of a stationary point and the investigation of the first approximation for stability is in general impossible.

In $[4,5]$ studied the conditions of stability zero solution for certain differential equation in the semi-linear case when the characteristic equation has roots of the form : $\lambda_{1}=i \lambda_{0}, \lambda_{0}>0$

and the others satisfying the property $\operatorname{Re} \lambda_{k}<-M, M>O, k=2, \ldots . ., n$. $[4,6]$ studied the same conditions to find the center of gravity for nonoutonomous quasi-linear differential equation of $\mathrm{n}$-th order .

In this paper, we study the conditions under which the zero solution is stable in the semi-linear case of differential equation which has the form :

$$
y^{\prime \prime \prime}+p_{1}(t) y^{\prime \prime}+p_{2}(t) y^{\prime}+p_{3}(t) y=h\left(t, y, y^{\prime}, y^{\prime \prime}\right)
$$

Where

$$
\begin{aligned}
& \mathrm{s}=1,2,3, w_{s}(t): \Delta \rightarrow C, q_{s} \in C, p_{s}=\pi^{s}\left[q_{s}+w_{s}(t)\right] \\
& t \in \Delta=[a, \infty), a \in N
\end{aligned}
$$

are continuos functions for all $\mathrm{s}$ and $\pi^{s}(t)$ times differentiable and $\alpha$ satisfies the following conditions :

$$
\begin{aligned}
& , \pi^{-2} \pi^{\prime}=0(1), \pi: \Delta \rightarrow(0, \infty) t \rightarrow \infty \\
& \qquad\left|h\left(t, y, y^{\prime}, y^{\prime \prime}\right)\right| \leq L^{*}\left[|y|+\left|y^{\prime}\right|+\left|y^{\prime \prime}\right|\right]^{1+\beta}, h: \Delta \times C^{n} \rightarrow C \\
& L^{*}: \Delta \rightarrow[0, \infty), \beta \geq 0 \quad \text { and the characteristic equation of }(1) \text { has roots, } \\
& \lambda_{1}=-\lambda_{2}=i \lambda_{0}, \lambda_{0}>0 \text { and the other root has the following property } \\
& \operatorname{Re} \lambda_{3}<-M, M>0 .
\end{aligned}
$$

\section{2- Definitions :}

Definition 1 [3] : The zero solution of the differential equation ( 1 ) is said to be stable as such that $\delta>0$ there exist $\forall \varepsilon>0$, if $t \rightarrow \infty$ the solution $\mathrm{y}=\mathrm{y}(\mathrm{t})$ of the differential equation (1) with the initial condition $|y(T)|<\delta$ satisfies the inequality $|y(t)|<\varepsilon, \forall \boldsymbol{t} \geq \boldsymbol{T}$

Definition 2 [3]: If the conditions of definition(1) are satisfied and then, zero solution of ( 1 ) is said to be asymptotically $\operatorname{Lim}_{t \rightarrow \infty} x(t)=O(1)$ stable . 


\section{3 - Helping Transformations}

In order to find the conditions under which the zero solution of differential equation ( 1 ) is stable, we use the following lemmas :

Lemma 1 [2] :

The transformation ;

$\mathrm{y}=\pi \cdot \mathrm{Z}_{1}$

$\mathrm{y}^{\prime}=\pi^{2} \cdot \mathrm{Z}_{2}$

$\mathrm{y}^{\prime \prime}=\pi^{3} \cdot \mathrm{Z}_{3}$ form :

transform the differential equation ( 1 ) to the differential system of the

$\mathrm{Z}_{1}^{\prime}=-\pi^{-1} \pi^{\prime} \mathrm{Z}_{1}+\pi \mathrm{Z}_{2}$

$Z_{2}^{\prime}=-2 \pi^{-1} \pi^{\prime} Z_{2}+\pi Z_{3}$

$\mathrm{Z}_{3}{ }^{\prime}=-\mathrm{p}_{3}(\mathrm{t}) \pi^{-2} \mathrm{Z}_{1}-\mathrm{p}_{2}(\mathrm{t}) \pi^{-1} \mathrm{Z}_{2}-\left(3 \pi^{-1} \pi^{\prime}+\mathrm{p}_{1}(\mathrm{t})\right) \mathrm{Z}_{3}+\mathrm{F}_{1}(\mathrm{t}, \mathrm{z})$ where

$\left|\mathrm{F}_{1}(\mathrm{t}, \mathrm{z})\right| \leq \mathrm{L}\left[\mathrm{Z}_{1}+\pi \mathrm{Z}_{2}+\pi^{2} \mathrm{Z}_{3}\right]^{1+\beta}, \mathrm{L}=\mathrm{L}^{*} \pi^{\beta-2}$

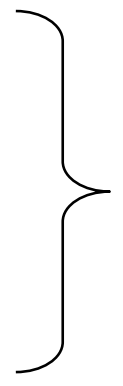




\section{Lemma $2[2]:$}

The transformation;

$\mathbf{X}=\mathbf{B Z}$

where

$$
B=\left[\begin{array}{crr}
\left(i \lambda_{0}\right)^{2}+q_{1}\left(i \lambda_{0}\right)+q_{2} & i \lambda_{0}+q_{1} & 1 \\
\left(-i \lambda_{0}\right)^{2}+q_{1}\left(i \lambda_{0}\right)+q_{2} & -i \lambda_{0}+q_{1} & 1 \\
1 & 0 & 0
\end{array}\right]
$$

$\mathrm{q}_{1}, \mathrm{q}_{2} \in \mathrm{C}, \quad \operatorname{det} \mathrm{B}=2 \mathrm{i} \lambda_{0}$

transform the differential system (3) to the system of the form :

$\left.\begin{array}{rl}\mathrm{X}_{1}{ }^{\prime} & =\pi\left[\mathrm{i} \lambda_{0} \mathrm{X}_{1}+\left(-\left(\mathrm{i} \lambda_{0}\right)^{3}-\mathrm{q}_{1}\left(\mathrm{i} \lambda_{0}\right)^{2}-\mathrm{q}_{2} \mathrm{i} \lambda_{0}-\mathrm{q}_{3}\right) \mathrm{X}_{3}\right]+\mathrm{F}_{2} \\ \mathrm{X}_{2}{ }^{\prime} & =\pi\left[\mathrm{q}_{1} \mathrm{X}_{1}+\left(-\mathrm{i} \lambda_{0}-\mathrm{q}_{1}\right) \mathrm{X}_{2}+\left(\left(\mathrm{i} \lambda_{0}\right)^{3}+\left(\mathrm{i} \lambda_{0}\right)^{2} \mathrm{q}_{1}+\mathrm{i} \lambda_{0} \mathrm{q}_{2}-\mathrm{q}_{3}\right) \mathrm{X}_{3}\right]+\mathrm{F}_{2} \\ X_{3}{ }^{\prime} & =\pi\left[\frac{1}{2 i \lambda_{0}} X_{1}-\frac{1}{2 i \lambda_{0}} X_{2}\right]\end{array}\right\}$

where ,

$$
\lim _{t \rightarrow \infty} W_{s}(t)=o(1), \quad \lim _{t \rightarrow \infty} \pi^{-2} \pi^{\prime}=o(1)
$$

$|F 2| \leq M L\left[\frac{\left.\left[-\pi+\pi^{2}\left(-i \lambda_{0}+q_{1}\right)\right] X_{1}+\left[\pi-\pi^{2}\left(i \lambda_{0}+q_{1}\right)\right] X_{2}+\left[-2 i \lambda_{0}+\pi^{2} 2\left(i \lambda_{0}\right)^{3}+2 q_{1}\left(i \lambda_{0}\right)^{2}+2 q_{2} i \lambda_{0}\right)\right] X_{3}}{-2 i \lambda_{0}}\right]^{1+\beta}$

$M>0$

Lemma 3 [2] :

By using the following transformation

$\left.\begin{array}{l}\mathrm{X}_{1}=\mathrm{y}_{1} \\ \mathrm{X}_{2}=\mathrm{y}_{2} \\ \mathrm{X}_{3}=\mathrm{ky}_{1}+\bar{k} \mathrm{y}_{2}+\mathrm{y}_{3}\end{array}\right\}$

where $\mathrm{k}, \bar{k} \in \mathrm{C}$

we transform the differential system ( 5 ) into the following differential system :

$$
\begin{aligned}
\mathrm{y}_{1}{ }^{\prime}= & \pi \square\left[\left(\mathrm{i} \lambda_{0}+\mathrm{c}_{1} \mathrm{k}\right) \mathrm{y}_{1}+\left(\mathrm{c}_{1} \bar{k}\right) \mathrm{y}_{2}+\mathrm{c}_{1} \mathrm{y}_{3}\right]+\mathrm{F}_{3} \\
\mathrm{y}_{2}{ }^{\prime}= & \pi \square\left[\left(\mathrm{q}_{1}+\mathrm{c}_{2} \mathrm{k}\right) \mathrm{y}_{1}+\left(-\mathrm{i} \lambda_{0}-\mathrm{q}_{1}+\mathrm{c}_{2} \overline{\mathrm{k}}\right) \mathrm{y}_{2}+\mathrm{c}_{2} \mathrm{y}_{3}\right]+\mathrm{F}_{3} \\
y_{3}{ }^{\prime}=\pi & {\left[\left[\frac{1}{2 i \lambda_{0}}-k\left(i \lambda_{0}+c_{1} k\right)-\bar{k}\left(q_{1}+c_{2} k\right)\right] y_{1}+\left[-\frac{1}{2 i \lambda_{0}}-k c_{1} \bar{k}-\bar{k}\left(-i \lambda_{0}-q_{1}+c_{2} \bar{k}\right)\right] y_{2}+\right.} \\
& {\left.\left[-k c_{1}-\bar{k} c_{2}\right] y_{3}\right]-F_{3}[k+\bar{k}] }
\end{aligned}
$$


$|F 3| \leq M L\left[\frac{\left(-\pi+\pi^{2}\left(-i \lambda_{0}+q_{1}\right)+k\left[-2 i \lambda_{0}+\pi^{2}\left(2\left(i \lambda_{0}\right)^{3}+2 q_{1}\left(i \lambda_{0}\right)^{2}+2 q_{2} i \lambda_{0}\right)\right.\right.}{-2 i \lambda_{0}} y_{1}+\right.$

$\frac{\left(\pi-\pi^{2}\left(-i \lambda_{0}+q_{1}\right)+\bar{k}\left[-2 i \lambda_{0}+\pi^{2}\left(2\left(i \lambda_{0}\right)^{3}+2 q_{1}\left(i \lambda_{0}\right)^{2}+2 q_{2} i \lambda_{0}\right)\right]\right.}{-2 i \lambda_{0}} y_{2}$

$\left.+\left[\frac{-2 i \lambda_{0}+\pi^{2}\left(2\left(i \lambda_{0}\right)^{3}+2 q_{1}\left(i \lambda_{0}\right)^{2}+2 q_{2} i \lambda_{0}\right)}{-2 i \lambda_{0}}\right] y_{3}\right]^{1+\beta}$

Now, we use the following lemma which leads to the auxiliary system :

\section{Lemma 4 [5] :}

the transform

$$
\left.\begin{array}{l}
\mathrm{y}_{1}=\mathrm{w}_{1}+\mathrm{bw}_{2}+\mathrm{b}_{3} \mathrm{w}_{3} \\
\mathrm{y}_{2}=\mathrm{bw}_{1}+\mathrm{w}_{2}+\mathrm{b}_{3} \mathrm{w}_{3} \\
\mathrm{y}_{3}=\mathrm{w}_{3}
\end{array}\right\}
$$

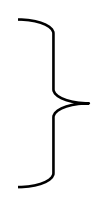

where $\mathrm{b}, \mathrm{b}_{3} \in \mathrm{C} \quad, \quad \mathrm{b} \neq \square \underline{ \pm 1}$

$$
\begin{aligned}
& w_{1}{ }^{\prime}=\frac{\pi}{1-b^{2}}\left[i \lambda_{0}+c_{1} k-b\left(q_{1}+c_{2} k\right)+b_{3}(b-1)\left[\frac{1}{2 i \lambda_{0}}-k\left(i \lambda_{0}+c_{1} k\right)\right.\right. \\
& \left.-\bar{k}\left(q_{1}+c_{2} k\right)\right]+b\left[c_{1} \bar{k}-b\left(-i \lambda_{0}-q_{1}+c_{2} \bar{k}\right)+b_{3}(b-1)\right. \\
& \left.\left.\left[-\frac{1}{2 i \lambda_{0}}-k c_{1} \bar{k}-\bar{k}\left(-i \lambda_{0}-q_{1}+c_{2} \bar{k}\right)\right]\right]\right] W_{1}+ \\
& \frac{\pi}{1-b^{2}}\left[b \left[i \lambda_{0}+c_{1} k-b\left(q_{1}+c_{2} k\right)+b_{3}(b-1)\left[\frac{1}{2 i \lambda_{0}}-k\left(i \lambda_{0}+c_{1} k\right)\right.\right.\right. \\
& \left.\left.-\bar{k}\left(q_{1}+c_{2} k\right)\right]\right]+c_{1} \bar{k}-b\left(-i \lambda_{0}-q_{1}+c_{2} \bar{k}\right)+b_{3}(b-1)\left[-\frac{1}{2 i \lambda_{0}}-k c_{1} \bar{k}-\right. \\
& \left.\left.\bar{k}\left(-i \lambda_{0}-q_{1}+c_{2} \bar{k}\right)\right]\right] W_{2}+ \\
& +\frac{\pi}{1-b^{2}}\left[b _ { 3 } \left[i \lambda_{0}+c_{1} k-b\left(q_{1}+c_{2} k\right)+b_{3}(b-1)\left[-k\left(i \lambda_{0}+c_{1} k\right)-\bar{k}\left(q_{1}+c_{2} k\right)\right]+c_{1} \bar{k}-\right.\right. \\
& \left.b\left(-i \lambda_{0}-q_{1}+c_{2} \bar{k}\right)+b_{3}(b-1)\left[-k c_{1} \bar{k}-\bar{k}\left(-i \lambda_{0}-q_{1}+c_{2} k\right)\right]\right]+c_{1}-b c_{2}+b_{3}(b-1) \\
& \left.\left[-k c_{1}-\bar{k} c_{2}\right]\right] W_{3}+\frac{1}{1-b^{2}} F_{4}\left[1-b-b_{3}(b-1)(k+\bar{k})\right]
\end{aligned}
$$




$$
\begin{aligned}
& w_{2}^{\prime}=\frac{\pi}{1-b^{2}}\left[-b\left(i \lambda_{0}+c_{1} k\right)+q_{1}+c_{2} k+b_{3}(b-1)\left[\frac{1}{2 i \lambda_{0}}-k\left(i \lambda_{0}+c_{1} k\right)\right.\right. \\
& \left.-\bar{k}\left(q_{1}+c_{2} k\right)\right]+b\left[-b c_{1} \bar{k}-i \lambda_{0}-q_{1}+c_{2} \bar{k}+b_{3}(b-1)\right. \\
& \left.\left.\left[-\frac{1}{2 i \lambda_{0}}-k c_{1} \bar{k}-\bar{k}\left(-i \lambda_{0}-q_{1}+c_{2} \bar{k}\right)\right]\right]\right] W_{1}+ \\
& \frac{\pi}{1-b^{2}}\left[b \left[-b\left(i \lambda_{0}+c_{1} k\right)+q_{1}+c_{2} k+b_{3}(b-1)\left[\frac{1}{2 i \lambda_{0}}-k\left(i \lambda_{0}+c_{1} k\right)\right.\right.\right. \\
& \left.\left.-\bar{k}\left(q_{1}+c_{2} k\right)\right]\right]-b c_{1} \bar{k}-i \lambda_{0}-q_{1}+c_{2} \bar{k}+b_{3}(b-1)\left[-\frac{1}{2 i \lambda_{0}}-k c_{1} \bar{k}-\right. \\
& \left.\left.\bar{k}\left(-i \lambda_{0}-q_{1}+c_{2} \bar{k}\right)\right]\right] W_{2} \\
& +\frac{\pi}{1-b^{2}}\left[b _ { 3 } \left[-b\left(i \lambda_{0}+c_{1} k\right)+q_{1}+c_{2} k+b_{3}(b-1)\left[-k\left(i \lambda_{0}+c_{1} k\right)\right.\right.\right. \\
& \left.-\bar{k}\left(q_{1}+c_{2} k\right)\right]-b c_{1} \bar{k}-i \lambda_{0}-q_{1}+c_{2} \bar{k}+b_{3}(b-1)\left[-k c_{1} \bar{k}-\right. \\
& \left.\left.\bar{k}\left(-i \lambda_{0}-q_{1}+c_{2} \bar{k}\right)\right]-b c_{1}+c_{2}+b_{3}(b-1)\left[-k c_{1}-\bar{k} c_{2}\right]\right] W_{3}+ \\
& +\frac{1}{1-b^{2}} F_{4}\left[1-b-b_{3}(b-1)(k+\bar{k})\right] \\
& w_{3}{ }^{\prime}=\pi\left[\frac{1}{2 i \lambda_{0}}-k\left(i \lambda_{0}+c_{1} k\right)-\bar{k}\left(q_{1}+c_{2} k\right)+b\left(-\frac{1}{2 i \lambda_{0}}-k c_{1} \bar{k}-\bar{k}\left(-i \lambda_{0}-q_{1}+c_{2} \bar{k}\right)\right)\right] W_{1}+ \\
& +\pi\left[b\left(\frac{1}{2 i \lambda_{0}}-k\left(i \lambda_{0}+c_{1} k\right)-\bar{k}\left(q_{1}+c_{2} k\right)\right)-\frac{1}{2 i \lambda_{0}}-k c_{1} \bar{k}-\bar{k}\left(-i \lambda_{0}-q_{1}+c_{2} \bar{k}\right)\right] W_{2} \\
& +\pi\left[b_{3}\left[-k\left(i \lambda_{0}+c_{1} k\right)-\bar{k}\left(q_{1}+c_{2} k\right)-k c_{1} \bar{k}-\bar{k}\left(-i \lambda_{0}-q_{1}+c_{2} \bar{k}\right)\right]-k c_{1}-\bar{k} c_{2}\right] w_{3}-F_{4}(k+\bar{k})
\end{aligned}
$$




$$
\begin{aligned}
& \mid F 4 \leq M L\left[\frac { 1 } { - 2 i \lambda _ { 0 } } \left[-\pi+\pi^{2}\left(-i \lambda_{o}+q_{1}\right)+k\left[-2 i \lambda_{0}+\pi^{2}\left(2\left(i \lambda_{0}\right)^{3}+2 q_{1}\left(i \lambda_{0}\right)^{2}+2 q_{2} i \lambda_{0}\right)\right]\right.\right. \\
& +b\left[\pi-\pi^{2}\left(i \lambda_{0}+q_{1}\right)+\bar{k}\left[-2 i \lambda_{0}+\pi^{2}\left(2\left(i \lambda_{0}\right)^{3}+2 q_{1}\left(i \lambda_{0}\right)^{2}+2 q_{2} i \lambda_{0}\right)\right]\right] W_{1} \\
& +\frac{1}{-2 i \lambda_{0}}\left[b\left[-\pi+\pi^{2}\left(-i \lambda_{0}+q_{1}\right)+k\left[-2 i \lambda_{0}+\pi^{2}\left(2\left(i \lambda_{0}\right)^{3}+2 q_{1}\left(i \lambda_{0}\right)^{2}+2 q_{2} i \lambda_{0}\right)\right]\right]\right. \\
& \left.+\pi-\pi^{2}\left(i \lambda_{0}+q_{1}\right)+\bar{k}\left[-2 i \lambda_{0}+\pi^{2}\left(2\left(i \lambda_{0}\right)^{3}+2 q_{1}\left(i \lambda_{0}\right)^{2}+2 q_{2} i \lambda_{0}\right)\right]\right] W_{2} \\
& +\frac{1}{-2 i \lambda_{0}}\left[b _ { 3 } \left[-2 i \lambda_{0} \pi^{2}+k\left[-2 i \lambda_{0}+\pi^{2}\left(2\left(i \lambda_{0}\right)^{3}+2 q_{1}\left(i \lambda_{0}\right)^{2}+2 q_{2} i \lambda_{0}\right)\right]\right.\right. \\
& \left.\left.+\bar{k}\left[-2 i \lambda_{0}+\pi^{2}\left(2\left(i \lambda_{0}\right)^{3}+2 q_{1}\left(i \lambda_{0}\right)^{2}+2 q_{2} i \lambda_{0}\right)\right]\right]-2 i \lambda_{0}+\pi^{2}\left(2\left(i \lambda_{0}\right)^{3}+2 q_{1}\left(i \lambda_{0}\right)^{2}+2 q_{2} i \lambda_{0}\right] W_{3}\right]
\end{aligned}
$$

\section{Lemma 5 [2] :}

the transform

$$
\left.\begin{array}{l}
\mathrm{w}_{1}=\mathrm{re}^{\mathrm{i} \theta} \\
\mathrm{w}_{2}=\mathrm{re}^{-\mathrm{i} \theta} \\
\mathrm{w}_{3}=-\mathrm{r}_{3}
\end{array}\right\}
$$

where $\quad \theta \in[0,2 \pi]$

transform ( 9 ) into the following differential system :

$$
\begin{aligned}
& r^{\prime}=\mu_{1} r+\mu_{2} r_{3}+\frac{e^{-i \theta}}{1-b^{2}} F_{5}\left[1-b-b_{3}(b-1)(k+\bar{k})\right] \\
& r^{\prime}=\mu_{1}^{*} r+\mu_{2}^{*} r_{3}+\frac{e^{i \theta}}{1-b^{2}} F_{5}\left[1-b-b_{3}(b-1)(k+\bar{k})\right] \\
& r_{3}^{\prime}=\mu_{1}^{* *} r+\mu_{2}^{* *} r_{3}-F_{5}[k+\bar{k}]
\end{aligned}
$$

where 


$$
\begin{aligned}
& \mu_{1}=\frac{\pi}{1-b^{2}}\left[i \lambda_{0}+c_{1} k-b\left(q_{1}+c_{2} k\right)+b_{3}(b-1)\left[\frac{1}{2 i \lambda_{0}}-k\left(i \lambda_{0}+c_{1} k\right)\right.\right. \\
& \left.-\bar{k}\left(q_{1}+c_{2} k\right)\right]+b\left[c_{1} \bar{k}-b\left(-i \lambda_{0}-q_{1}+c_{2} \bar{k}\right)+b_{3}(b-1)\left[-\frac{1}{2 i \lambda_{0}}-k c_{1} \bar{k}\right.\right. \\
& \left.\left.-\bar{k}\left(-i \lambda_{0}-q_{1}+c_{2} \bar{k}\right)\right]\right] \\
& +\frac{\pi}{1-b^{2}}\left[b \left[i \lambda_{0}+c_{1} k-b\left(q_{1}+c_{2} k\right)+b_{3}(b-1)\left[\frac{1}{2 i \lambda_{0}}-k\left(i \lambda_{0}+c_{1} k\right)\right.\right.\right. \\
& \left.\left.-\bar{k}\left(q_{1}+c_{2} k\right)\right]\right]+c_{1} \bar{k}-b\left(i \lambda_{0}-q_{1}+c_{2} \bar{k}\right)+b_{3}(b-1)\left[-\frac{1}{2 i \lambda_{0}}-k c_{1} \bar{k}-\right. \\
& \left.\left.-\bar{k}\left(-i \lambda_{0}-q_{1}+c_{2} \bar{k}\right)\right]\right] e^{-2 i \theta} \\
& \mu_{2}=-\frac{\pi e^{-i \theta}}{1-b^{2}} b_{3}\left[i \lambda_{0}+c_{1} k-b\left(q_{1}+c_{2} k\right)+b_{3}(b-1)\left[-k\left(i \lambda_{0}+c_{1} k\right)-\bar{k}\left(q_{1}+\right.\right.\right. \\
& \left.\left.\left.c_{2} k\right)\right]+c_{1} \bar{k}-b\left(-i \lambda_{0}-q_{1}+c_{2} \bar{k}\right)+b_{3}(b-1)\left[-k c_{1} \bar{k}-\bar{k}\left(-i \lambda_{0}-q_{1}+c_{2} \bar{k}\right)\right]\right] \\
& -\frac{\pi e^{-i \theta}}{1-b^{2}}\left[c_{1}-b c_{2}+b_{3}(b-1)\left[-k c_{1}-\bar{k} c_{2}\right]\right] . \\
& \mu_{1}^{*}=\frac{\pi}{1-b^{2}}\left[-b\left(i \lambda_{0}+c_{1} k\right)+q_{1}+c_{2} k+b_{3}(b-1)\left[\frac{1}{2 i \lambda_{0}}-k\left(i \lambda_{0}+c_{1} k\right)\right.\right. \\
& \left.-\bar{k}\left(q_{1}+c_{2} k\right)\right]+b\left[-b c_{1} \bar{k}-i \lambda_{0}-q_{1}+c_{2} \bar{k}+b_{3}(b-1)\left[-\frac{1}{2 i \lambda_{0}}-k c_{1} \bar{k}\right.\right. \\
& \left.\left.-\bar{k}\left(-i \lambda_{0}-q_{1}+c_{2} \bar{k}\right)\right]\right] e^{2 i \theta} \\
& +\frac{\pi}{1-b^{2}}\left[b \left[-b\left(i \lambda_{0}+c_{1} k\right)+q_{1}+c_{2} k+b_{3}(b-1)\left[\frac{1}{2 i \lambda_{0}}-k\left(i \lambda_{0}+c_{1} k\right)\right.\right.\right. \\
& \left.\left.-\bar{k}\left(q_{1}+c_{2} k\right)\right]\right]-b c_{1} \bar{k}-i \lambda_{0}-q_{1}+c_{2} \bar{k}+b_{3}(b-1)\left[-\frac{1}{2 i \lambda_{0}}-k c_{1} \bar{k}-\right. \\
& \left.\left.\bar{k}\left(-i \lambda_{0}-q_{1}+c_{2} \bar{k}\right)\right]\right]
\end{aligned}
$$




$$
\begin{aligned}
& \mu_{2}^{*}=-\frac{\pi e^{i \theta}}{1-b^{2}} b_{3}\left[-b\left(i \lambda_{0}+c_{1} k\right)+q_{1}+c_{2} k+b_{3}(b-1)\left[-k\left(i \lambda_{0}+c_{1} k\right)-\bar{k}\left(q_{1}+\right.\right.\right. \\
& \left.\left.\left.c_{2} k\right)\right]-b c_{1} \bar{k}-i \lambda_{0}-q_{1}+c_{2} \bar{k}+b_{3}(b-1)\left[-k c_{1} \bar{k}-\bar{k}\left(-i \lambda_{0}-q_{1}+c_{2} \bar{k}\right)\right]\right] \\
& -\frac{\pi e^{i \theta}}{1-b^{2}}\left[-b c_{1}+c_{2}+b_{3}(b-1)\left[-k c_{1}-c_{2} \bar{k}\right]\right] \\
& \mu_{1}^{* *}=-\pi\left[\frac{1}{2 i \lambda_{0}}-k\left(i \lambda_{0}+c_{1} k\right)-\bar{k}\left(q_{1}+c_{2} k\right)+b\left[-\frac{1}{2 i \lambda_{0}}-k c_{1}-\right.\right. \\
& \left.\left.-\bar{k}\left(-i \lambda_{0}-q_{1}+c_{2} \bar{k}\right)\right]\right] e^{i \theta} \\
& \left.-\pi\left[b\left[\frac{1}{2 i \lambda_{0}}-k\left(i \lambda_{0}+c_{1} k\right)-\bar{k}\left(q_{1}+c_{2} k\right)\right]-\frac{1}{2 i \lambda_{0}}-k c_{1} \bar{k}-\bar{k}\left(-i \lambda_{0}-q_{1}+c_{2} \bar{k}\right)\right]\right] e^{-i \theta} \\
& \mu_{2}^{* *}=-\pi b_{3}\left[-k\left(i \lambda_{0}+c_{1} k\right)-\bar{k}\left(q_{1}+c_{2} k\right)-k c_{1} \bar{k}-\bar{k}\left(-i \lambda_{0}-q_{1}+c_{2} \bar{k}\right)\right] \\
& -\pi\left[-k c_{1}-\bar{k} c_{2}\right] \\
& |F 5| \leq M L\left[\frac { 1 } { - 2 i \lambda _ { 0 } } \left[-\pi+\pi^{2}\left(-i \lambda_{0}+q_{1}\right)+k\left[-2 i \lambda_{0}+\pi^{2}\left(2\left(i \lambda_{0}\right)^{3}+2 q_{1}\left(i \lambda_{0}\right)^{2}+2 q_{2} i \lambda_{0}\right)\right]\right.\right. \\
& \left.+b\left[\pi-\pi^{2}\left(i \lambda_{0}+q_{1}\right)+\bar{k}\left[-2 i \lambda_{0}+\pi^{2}\left(2\left(i \lambda_{0}\right)^{3}+2 q_{1}\left(i \lambda_{0}\right)^{2}+2 q_{2} i \lambda_{0}\right)\right]\right]\right] e^{i \theta} \\
& +\left[b\left[-\pi+\pi^{2}\left(i \lambda_{0}+q_{1}\right)+k\left[-2 i \lambda_{0}+\pi^{2}\left(2\left(i \lambda_{0}\right)^{3}+2 q_{1}\left(i \lambda_{0}\right)^{2}+2 q_{2} i \lambda_{0}\right)\right]\right]\right. \\
& \left.\left.+\pi-\pi^{2}\left(i \lambda_{0}+q_{1}\right)+\bar{k}\left[-2 i \lambda_{0}+\pi^{2}\left(2\left(i \lambda_{0}\right)^{3}+2 q_{1}\left(i \lambda_{0}\right)^{2}+2 q_{2} i \lambda_{0}\right)\right]\right] e^{i \theta}\right) r \\
& -\frac{1}{-2 i \lambda_{0}}\left[b_{3}\left[-2 i \lambda_{0} \pi^{2}+[k+\bar{k}]\left(-2 i \lambda_{0}+\pi^{2}\left(2\left(i \lambda_{0}\right)^{3}+2 q_{1}\left(i \lambda_{0}\right)^{2}+2 q_{2} i \lambda_{0}\right)\right)\right]\right. \\
& \left.-2 i \lambda_{0}+\pi^{2}\left(2\left(i \lambda_{0}\right)^{3}+2 q_{1}\left(i \lambda_{0}\right)^{2}+2 q_{2} i \lambda_{0}\right] r_{3}\right]^{1+\beta}
\end{aligned}
$$

\section{4 - Fundamental results :}

\section{Theorem :}

In the equation ( 1 ) if :

$$
\begin{gathered}
, h: \Delta \times C^{n} \rightarrow C, \quad p_{s}: \Delta=[a, \infty) \rightarrow C 1- \\
\left|h\left(t, y, y^{\prime}, y^{\prime \prime}\right)\right| \leq L^{*}\left[|y|+\left|y^{\prime}\right|+\left|y^{\prime \prime}\right|\right]^{1+\beta} \\
L^{*}: \Delta \rightarrow[0, \infty) \quad, \quad \beta \geq 0 \\
2-\quad \lim _{t \rightarrow \infty} \pi^{-2} \pi^{\prime}=o(1) \quad, \quad \lim _{t \rightarrow \infty} W_{s}(t)=o(1)
\end{gathered}
$$


and

a $-\quad t \rightarrow \infty$ as $\quad \int_{T}^{t} \operatorname{Re} \mu d t \rightarrow-\infty$ if

b - $\quad e^{\int_{T} \operatorname{Re} \mu d t} \int_{T}^{t} \mu_{1} e^{-\int_{T}^{t} \operatorname{Re} \mu d t} d t=o(1), t \rightarrow \infty$

then the zero solution of ( 1 ) is stable

c- as $\quad \int_{T}^{t} \operatorname{Re} \mu d t \rightarrow \infty$ if $t \rightarrow \infty$

then the zero solution of ( 1 ) is unstable

\section{proof}

on applying the transformation (2), (4), (8) and (10) into (1) we get the auxiliary system :

$$
\begin{aligned}
& r^{\prime}=\mu_{1} r+\mu_{2} \xi_{3}+\frac{e^{-i \theta}}{1-b^{2}} F_{6}\left[1-b-b_{3}(b-1)(k+\bar{k})\right] \\
& r^{\prime}=\mu_{1}^{*} \xi_{2}+\mu_{2}^{*} \xi_{2}+\frac{e^{-i \theta}}{1-b^{2}} F_{6}\left[1-b-b_{3}(b-1)(k+\bar{k})\right] \\
& r_{3}^{\prime}=\mu_{1}^{* *} \xi_{1}+\mu_{2}^{* *} r_{3}-F_{6}[k+\bar{k}]
\end{aligned}
$$

is an arbitrary variant function and it is continuous $\xi=\xi(t)$ where for all $\quad t \geq T \quad$ the auxiliary system ( 12 ) solved by the method Variation of parameters " [ 1 ]

$$
\begin{aligned}
& |r| \leq e^{\int_{T}^{t} \operatorname{Re} \mu_{1} d t}\left[r(T)+\int_{T}^{t}\left(\mu_{2} \xi_{3}+\frac{e^{-i \theta}}{1-b^{2}} F_{6}\left[1-b-b_{3}(b-1)(k+\bar{k})\right]\right) e^{-\int \operatorname{t} \operatorname{Re} \mu_{1}(t) d t} d t\right] \\
& |r| \leq e^{\int_{T}^{t} \operatorname{Re} \mu_{1}^{*} d t}\left[r(T)+\int_{T}^{t}\left(\mu_{2}^{*} \xi_{2}+\frac{e^{i \theta}}{1-b^{2}} F_{6}\left[1-b-b_{3}(b-1)(k+\bar{k})\right]\right) e^{-\iint_{T}^{t} \operatorname{Re} \mu_{1}^{*}(t) d t} d t\right] \\
& \left|r_{3}\right| \leq e^{\int_{T}^{t} \operatorname{Re} \mu_{2}^{* *} d t}\left[r(T)+\int_{T}^{t}\left(\mu_{1}^{* *} \xi_{1}-F_{6}[(k+\bar{k})] e^{-\iint_{T}^{t} \operatorname{Re} \mu_{2}^{* *}(t) d t} d t\right]\right.
\end{aligned}
$$

Now it is clear if

$$
1-e^{\int_{T}^{t} \operatorname{Re} \mu d t}=0,2-e^{\int^{t} \operatorname{Re} \mu^{*} d t}=0,3-e^{\int^{t} \operatorname{Re} \mu^{* *} d t}=0
$$

then the zero solution of equation ( 1 ) is stable

To explain our fundamental results the following example is given: 
$y^{\prime \prime \prime}+\pi\left[(1-i)+(\operatorname{lnt})^{-1}(2+3 i)\right] y^{\prime \prime}+\pi^{2}\left[4+(\operatorname{lnt})^{-2}(1-4 i)\right] y^{\prime}+\pi^{3}\left[(4-4 i)+t^{-1}(3-6 i)\right] y$ $=L^{*}\left[|y|+\left|y^{\prime}\right|+\left|y^{\prime \prime}\right|\right]^{1+\beta}$.............. (A ) , $\quad \beta \in[0, \infty)$

The characteristic equation to homogeneous part of equation ( $\mathrm{A}$ )

Contains roots form : $\quad \lambda_{1}=2 \mathrm{i}, \quad \lambda_{2}=-2 \mathrm{i}, \quad \lambda_{3}=-1+\mathrm{i}$
when
$\lim _{t \rightarrow \infty} \pi^{-2} \pi^{\prime}=o(1) \quad, \quad \lim _{t \rightarrow \infty} W_{s}(t)=o(1)$
$\mathrm{b}=1+\mathrm{i} \quad, \quad \mathrm{b}_{3}=1, \quad \mathrm{k}=2+\mathrm{i}, \quad \bar{k}=2-\mathrm{i}$
then $\pi=-t^{-1 / 2} \quad$ or $\quad \pi=e^{t}$ for example if $\quad \lim _{t \rightarrow \infty} \pi^{-2} \pi^{\prime}=o(1)$

on applying the transformation (2), (4) (8) and (10) into ( A ) we get the following table:

\begin{tabular}{|c|c|c|c|c|c|c|c|c|}
\hline & \multicolumn{4}{|c|}{$\pi=-\mathrm{t}^{-1 / 2}$} & \multicolumn{4}{|l|}{$\pi=\mathrm{e}^{\mathrm{t}}$} \\
\hline & $\theta=0$ & $\theta=30$ & $\theta=45$ & $\theta=60$ & $\theta=0$ & $\theta=\mathbf{3 0}$ & $\theta=45$ & $\theta=60$ \\
\hline $\int_{T}^{t} \operatorname{Re} \mu_{1} \mathrm{dt}$ & $\infty$ & $-\infty$ & $-\infty$ & $-\infty$ & $-\infty$ & $\infty$ & $\infty$ & $\infty$ \\
\hline $\int_{T}^{t} \operatorname{Re} \mu_{1}^{*} \mathrm{dt}$ & $\infty$ & $\infty$ & $\infty$ & $\infty$ & $-\infty$ & $-\infty$ & $-\infty$ & $-\infty$ \\
\hline \multirow[t]{2}{*}{$\int_{T}^{t} \operatorname{Re} \mu_{2}^{* * *} \mathrm{dt}$} & \multicolumn{4}{|l|}{$\infty$} & \multicolumn{4}{|l|}{$-\infty$} \\
\hline & \multicolumn{4}{|c|}{ unstable } & stable & unstable & unstable & unstable \\
\hline
\end{tabular}




\section{REFERENCES}

[1] EL slog s ,L .E. (1977) Differential Equations and Calculus of Variation, India .

[2] Malkin, I.G., (1966) .Theory of Stability of Motion , Moscow.Nauk publishing co. Moscow. Inc, USSR.

[3] Miller, R. K.; Michel A. N., (1982) . Ordinary Differential Equations, London.Academic press,Inc.

[4] Thanoon, T.Y, (1990). Center of Gravity of non-Autonmous Nonlinaer Differential System in Some Critical Cases. Unpublished Ph.D. Thesis, Odessa, USSR .

[5] Thanoon, T.Y, AL-Katib A.Z., (1999). "The Finding Conditions of Stability Trivial Solution for Certain Differential Equation in the Semi-liner Case , J.Edu . Sci., Vol(37) , PP. 88-96 .

[6] Thanoon, T.Y, Etewi J. R., (2001). On the Center of Gravity for Quasi - linear Differential Equation of Order $\mathrm{n}$ in the Critical Case , J.Edu. Sci., Vol.(12), PP. 82 - 90 . 